\title{
On the regulation of the energy of elastic joint robots: excitation and damping of oscillations
}

\author{
Gianluca Garofalo, Johannes Englsberger and Christian Ott
}

\begin{abstract}
The paper presents a new control law for elastic joint robots that allows to regulate the energy stored in the system to a desired value. Being able to either remove energy from the system or inject it, oscillations can be both damped out and induced. Therefore the control law can be used for asymptotic regulation to a desired configuration and (in case of additional constraints) generation of asymptotically stable limit cycles. Compared to other methods, we can formally guarantee the previous property keeping at the same time the control law simple and easy to implement. Furthermore, using the energy stored by the intrinsic elastic elements in the joints, high energy efficiency is achieved. Simulations and experiments are also provided, in order to further validate the theoretical results.
\end{abstract}

\section{INTRODUCTION}

Elastic joint robots have been lately the focus of numerous researchers because of the capability to perform highly dynamical, explosive and cyclic motions. The energy stored in the elastic elements can be exploited to increase velocity and reducing the effort of the motors. However, the mechanical design of such systems introduces often additional nonlinearities in the system dynamics which, along with the underactuation problem, make the controller design more challenging. For these reasons, traditionally, the inevitable elasticity of the joint has been seen as a disturbance to minimize both in the mechanical and control design phases.

The focus of our research is to find new and efficient methods to produce periodic motions that can be used in general and in particular for locomotion [1], [2], [3], [4], [5]. Nevertheless, the contribution of this work goes actually in two directions.

Firstly, we provide what is to our knowledge the first control law that can directly induce oscillations for an elastic joint robot with no strong simplifying hypothesis. We pursue the idea already presented in our previous works of controlling the energy in order to produce a periodic motion. As it was mentioned there, the energy of the elastic elements can be used to sustain the oscillation. This is exactly what we achieve here in the nominal case. As we will show later, it is possible to obtain a periodic motion of the link while keeping the motor still. Basically, the motor pumps in the required energy and then stops, letting the spring sustain the oscillation. The method presented here solves the problem of orbital stabilization for elastic joint robots without considering the rigid case as an intermediate step, hence without requiring computation of higher order derivatives [6], [7], filtering and state estimation, nor single

The authors are with the Institute of Robotics and Mechatronics, German Aerospace Center (DLR), Wessling, Germany. gianluca.garofalo@dlr.de

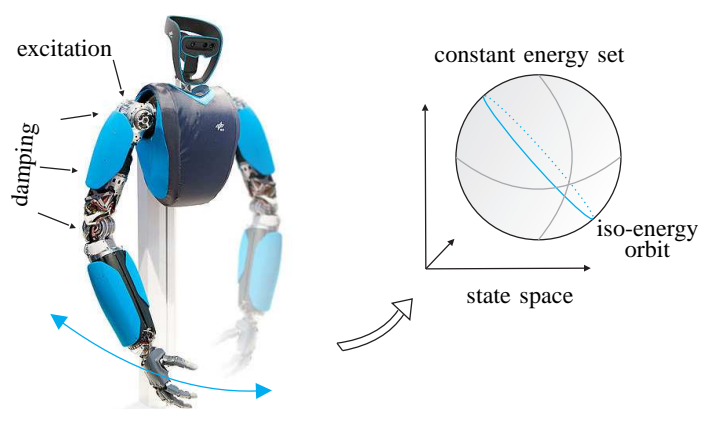

Fig. 1. The DLR Hand Arm System (HASy), used to validate the theoretical results of the paper, and conceptual representation of the effect of the controller in the state space.

perturbation hypothesis which overcome the problem using one of the motor side states as a virtual input [8]. Although, at this stage, the proof allows to conclude that the oscillation is due to the existence of an asymptotically stable limit cycle only for a single joint system. In this sense, what is presented here, can be seen as a first step towards the extension to the elastic case of our previous results [2], where (constraining the system to evolve on a 1 - dimensional submanifold) no restriction on the number of joints of the fully actuated robot was made.

Secondly, the same control law can be used to regulate the robot to a desired configuration, independently on the number of joints. Compared to classic results for elastic joint robots [9], [10] also a scaling of the torque produced by the elastic elements is included. On the other hand, recently other approaches have been suggested where a control strategy based on the energy of the system was used to damp out the oscillations of an elastic joint robot [11]. In that work the authors could not provide a formal analysis, nor ensure the recover of the configuration where the robot was previously in. With the work presented in this paper we can instead, at the price of requiring a small knowledge of the system, guarantee asymptotic properties and keep at the same time the control law simple and easy to implement.

The paper is organized as follows: in Section II we provide the dynamic equations describing the system and all the assumptions required to obtain the main result of the paper, which is presented in Section III. Section IV collects simulation and experimental results obtained implementing our control law for the DLR Hand Arm System [12] shown in Fig. 1, demonstrating both the possible application as a way to excite or damp oscillations. Section V concludes our work with a short discussion and outline of future work. 


\section{Prerequisites}

In what follows we will consider underactuated robots with elastic joints that, due to the assumptions collected in [13], can be modeled as

$$
\begin{aligned}
M(\boldsymbol{q}) \ddot{\boldsymbol{q}}+\boldsymbol{C}(\boldsymbol{q}, \dot{\boldsymbol{q}}) \dot{\boldsymbol{q}}+\boldsymbol{g}(\boldsymbol{q}) & =\tau(\phi) \\
\boldsymbol{B} \ddot{\boldsymbol{\theta}}+\tau(\phi) & =\boldsymbol{u},
\end{aligned}
$$

where $\boldsymbol{\theta}, \boldsymbol{q}, \dot{\boldsymbol{\theta}}, \dot{\boldsymbol{q}} \in \mathbb{R}^{n}$ constitute together the state of the system being $\boldsymbol{\theta}$ the motor position and $\boldsymbol{q}$ the link position, $\boldsymbol{u} \in \mathbb{R}^{n}$ is the input to the system provided by the motors, $\boldsymbol{M}(\boldsymbol{q}) \in \mathbb{R}^{n \times n}$ is the positive definite mass matrix, $\boldsymbol{C}(\boldsymbol{q}, \dot{\boldsymbol{q}}) \in$ $\mathbb{R}^{n \times n}$ is the Coriolis matrix, $\boldsymbol{g}(\boldsymbol{q})=\nabla_{\boldsymbol{q}} U_{g}(\boldsymbol{q}) \in \mathbb{R}^{n}$ is the gravity torque vector ${ }^{1}$ and $U_{g}(\boldsymbol{q})$ the gravitational potential, $\boldsymbol{\phi}=\boldsymbol{\theta}-\boldsymbol{q}$ collects the deflections of the springs which connect the motors to the links, $\tau(\phi)=\nabla_{\phi} U_{k}(\phi) \in \mathbb{R}^{n}$ is the torque produced by the springs and $U_{k}(\phi)$ the correspondent potential function.

In case the robot contains not only rotational joints, we will always consider the subset $\mathbb{Q} \subset \mathbb{R}^{n}$ in which all the prismatic joints keep bounded. In this case it is well known that the following proposition holds [14]

Proposition 1: For every matrix norm there exists a $\beta$ such that

$$
\left\|\frac{\partial^{2} U_{g}(\boldsymbol{q})}{\partial \boldsymbol{q}^{2}}\right\|<\beta, \forall \boldsymbol{q} \in \mathbb{Q} .
$$

Assumptions: In addition, the following properties are assumed

- $U_{k}(\phi)$ is a nonlinear scalar function

- $\alpha_{1}\|\xi\|^{2}<\xi^{T} \frac{\partial^{2} U_{k}(\boldsymbol{\phi})}{\partial \phi^{2}} \boldsymbol{\xi}<\alpha_{2}\|\xi\|^{2}, \forall \boldsymbol{\xi} \in \mathbb{Q}$

- $\beta<\alpha_{1}$

where, since $\beta$ must be positive, clearly also $\alpha_{1}, \alpha_{2}>0$. Assumption (A3) in particular, as explained in [10], states that the robot should be designed properly. Namely, the binding forces should grow faster than the diverging forces between $\boldsymbol{\theta}$ and $\boldsymbol{q}$, meaning that the joint stiffness should be sufficiently high to prevent the robot from falling down under the load of its own weight.

In [10] the problem of constructing an online gravity compensation for an elastic joint robot based on the motor position was solved using a static mapping between motor and link positions. The basic idea is to find, for any motor position, the link position which allows the elastic elemnts in the joints to compensate for the gravity term. Defining $U(\boldsymbol{\theta}, \boldsymbol{q})$ as $U(\boldsymbol{\theta}, \boldsymbol{q})=U_{g}(\boldsymbol{q})+U_{k}(\boldsymbol{\theta}-\boldsymbol{q})$, then assumptions (A2), (A3) allow to conclude that the function $\overline{\boldsymbol{q}}: \mathbb{R}^{n} \rightarrow \mathbb{R}^{n}$ defined by the implicit equation

$$
\left.\nabla_{\boldsymbol{q}} U(\boldsymbol{\theta}, \boldsymbol{q})\right|_{(\boldsymbol{\theta}, \overline{\boldsymbol{q}}(\boldsymbol{\theta}))}=\boldsymbol{g}(\overline{\boldsymbol{q}}(\boldsymbol{\theta}))-\boldsymbol{\tau}(\boldsymbol{\theta}-\overline{\boldsymbol{q}}(\boldsymbol{\theta}))=\mathbf{0},
$$

$\forall \boldsymbol{\theta} \in \mathbb{R}^{n}$, exists, is unique and is a diffeomorphism [9]. Therefore, the desired configuration of the robot can be

\footnotetext{
${ }^{1}$ With the symbol $\nabla_{x}(\cdot)$ we are simply indicating $\left(\frac{\partial(\cdot)}{\partial x}\right)^{T}$ in order to ease the notation.
}

indifferently given in terms of motor or link position, since the two are uniquely related through the function $\overline{\boldsymbol{q}}$.

Additionally, given the assumptions (A2), (A3), the "energy-like" function

$$
H(\boldsymbol{\theta}, \boldsymbol{q}, \dot{\boldsymbol{\theta}}, \dot{\boldsymbol{q}})=\frac{1}{2} \dot{\boldsymbol{q}}^{T} \boldsymbol{M}(\boldsymbol{q}) \dot{\boldsymbol{q}}+U(\boldsymbol{\theta}, \boldsymbol{q})-U(\boldsymbol{\theta}, \overline{\boldsymbol{q}}(\boldsymbol{\theta})),
$$

is a positive semidefinite function of the state and in particular

$$
H(\boldsymbol{\theta}, \boldsymbol{q}, \dot{\boldsymbol{\theta}}, \dot{\boldsymbol{q}})=0 \Longleftrightarrow \boldsymbol{q}=\overline{\boldsymbol{q}}(\boldsymbol{\theta}), \dot{\boldsymbol{q}}=\mathbf{0}
$$

$\forall \boldsymbol{\theta} \in \mathbb{Q}$. The proof of this property can be easily derived using Lemma 2 reported in the appendix.

\section{MaIn Result}

In this section we present the main result of the paper. For the sake of clarity we first report a result about the invariance principle [15, Lemma 2], which will be used later in the proof.

Lemma 1: Let $€$ be the positive limit set ( $\omega$ - limit set) of a bounded solution of a dynamical system. Given some technical assumptions [15], a $C^{1}$ scalar function of the state $W$ such that $\dot{W} \geq 0$ along the flow of the system and indicating with $E$ the set where $\dot{W}=0$, then $€$ and $E$ have nonempty intersection. Moreover if $€$ does not reduce to a single point, then $€ \cap E$ does not contain any stable equilibrium point.

Theorem 1 (Main result): Given the system (1) and the assumptions (A1) - (A3), let $K_{H}$ be a positive scalar, $\boldsymbol{K}_{\boldsymbol{\theta}}$ and $\boldsymbol{D}_{\boldsymbol{\theta}}$ two symmetric and positive definite matrices and finally let us denote with the tilde the difference between a variable and the correspondent desired constant value (indicated with the subscript " $d$ "), then the control input

$$
\boldsymbol{u}=\boldsymbol{\tau}(\boldsymbol{\phi})-K_{H} \tilde{H}(\boldsymbol{\theta}, \boldsymbol{q}, \dot{\boldsymbol{\theta}}, \dot{\boldsymbol{q}})(\boldsymbol{\tau}(\boldsymbol{\phi})-\boldsymbol{g}(\overline{\boldsymbol{q}}(\boldsymbol{\theta})))-\boldsymbol{K}_{\boldsymbol{\theta}} \tilde{\boldsymbol{\theta}}-\boldsymbol{D}_{\boldsymbol{\theta}} \dot{\boldsymbol{\theta}}
$$

will produce a closed loop system that will always reach the set

$$
\Omega=\left\{(\boldsymbol{\theta}, \boldsymbol{q}, \dot{\boldsymbol{\theta}}, \dot{\boldsymbol{q}}) \mid \boldsymbol{\theta}=\boldsymbol{\theta}_{d}, \dot{\boldsymbol{\theta}}=\mathbf{0}, H\left(\boldsymbol{\theta}_{d}, \boldsymbol{q}, \mathbf{0}, \dot{\boldsymbol{q}}\right)=H_{d}\right\}
$$

and therefore:

(a) for $H_{d}=0$ has an asymptotically stable equilibrium point in $(\boldsymbol{\theta}, \boldsymbol{q}, \dot{\boldsymbol{\theta}}, \dot{\boldsymbol{q}})=\chi_{0}$, where $\boldsymbol{\chi}_{0}=\left(\boldsymbol{\theta}_{d}, \overline{\boldsymbol{q}}\left(\boldsymbol{\theta}_{d}\right), \mathbf{0}, \mathbf{0}\right)$

(b) for $H_{d}>0$ and $n=1$ has an unstable equilibrium point in $(\theta, q, \dot{\theta}, \dot{q})=\chi_{0}$, where $\chi_{0}=\left(\theta_{d}, \bar{q}\left(\theta_{d}\right), 0,0\right)$ and an asymptotically stable limit cycle defined by $\Omega=$ $\left\{(\theta, q, \dot{\theta}, \dot{q}) \mid \theta=\theta_{d}, \dot{\theta}=0, H\left(\theta_{d}, q, 0, \dot{q}\right)=H_{d}\right\}$.

Proof: The proof is a bit cumbersome and therefore split in multiple parts. The goal is to show that $H \rightarrow H_{d}$ always. First we consider a Lyapunov function that shows the stability of the invariant set $\Omega$ of the closed loop system. Using LaSalle's invariance principle [16] we will then prove that the equilibrium point $\chi_{0}$ and the set $\Omega$ are the only positive limit sets for the solutions of (1) with the control law (6). Finally, using Lemma 1 and depending on the value of $H_{d}$, the results (a) and (b) naturally arise. 
Stability: The $C^{1}$ function of the state

$$
V(\boldsymbol{\theta}, \boldsymbol{q}, \dot{\boldsymbol{\theta}}, \dot{\boldsymbol{q}})=\frac{1}{2} K_{H} \tilde{H}^{2}(\boldsymbol{\theta}, \boldsymbol{q}, \dot{\boldsymbol{\theta}}, \dot{\boldsymbol{q}})+\frac{1}{2} \tilde{\boldsymbol{\theta}}^{T} \boldsymbol{K}_{\boldsymbol{\theta}} \tilde{\boldsymbol{\theta}}+\frac{1}{2} \dot{\boldsymbol{\theta}}^{T} \boldsymbol{B} \dot{\boldsymbol{\theta}}
$$

is such that

$$
\begin{cases}V(\boldsymbol{\theta}, \boldsymbol{q}, \dot{\boldsymbol{\theta}}, \dot{\boldsymbol{q}})=0 & \forall(\boldsymbol{\theta}, \boldsymbol{q}, \dot{\boldsymbol{\theta}}, \dot{\boldsymbol{q}}) \in \Omega \\ V(\boldsymbol{\theta}, \boldsymbol{q}, \dot{\boldsymbol{\theta}}, \dot{\boldsymbol{q}})>0 & \forall(\boldsymbol{\theta}, \boldsymbol{q}, \dot{\boldsymbol{\theta}}, \dot{\boldsymbol{q}}) \notin \Omega\end{cases}
$$

and therefore a candidate Lyapunov function. Before computing its derivative along the flow of the closed loop system, the same needs to be done for (4). To this end, it is useful to first notice that

$$
\begin{aligned}
\nabla_{\boldsymbol{\theta}} U(\boldsymbol{\theta}, \overline{\boldsymbol{q}}(\boldsymbol{\theta})) & =\left.\nabla_{\boldsymbol{\theta}} U(\boldsymbol{\theta}, \boldsymbol{q})\right|_{(\boldsymbol{\theta}, \overline{\boldsymbol{q}}(\boldsymbol{\theta}))}= \\
& =\boldsymbol{\tau}(\boldsymbol{\theta}-\overline{\boldsymbol{q}}(\boldsymbol{\theta})),
\end{aligned}
$$

since the term depending on $\left.\nabla_{\boldsymbol{q}} U(\boldsymbol{\theta}, \boldsymbol{q})\right|_{(\boldsymbol{\theta}, \overline{\boldsymbol{q}}(\boldsymbol{\theta}))}$ disappears due to (3). From (3) it also follows that $\boldsymbol{g}(\overline{\boldsymbol{q}}(\boldsymbol{\theta}))=\boldsymbol{\tau}(\boldsymbol{\theta}-\overline{\boldsymbol{q}}(\boldsymbol{\theta}))$, leading to

$$
\nabla_{\boldsymbol{\theta}} U(\boldsymbol{\theta}, \overline{\boldsymbol{q}}(\boldsymbol{\theta}))=\boldsymbol{g}(\overline{\boldsymbol{q}}(\boldsymbol{\theta})),
$$

which finally is used to obtain respectively

$$
\begin{aligned}
& \dot{H}(\boldsymbol{\theta}, \boldsymbol{q}, \dot{\boldsymbol{\theta}}, \dot{\boldsymbol{q}})=\dot{\boldsymbol{\theta}}^{T}(\boldsymbol{\tau}(\boldsymbol{\phi})-\boldsymbol{g}(\overline{\boldsymbol{q}}(\boldsymbol{\theta}))) \\
& \dot{V}(\boldsymbol{\theta}, \boldsymbol{q}, \dot{\boldsymbol{\theta}}, \dot{\boldsymbol{q}})=-\dot{\boldsymbol{\theta}}^{T} \boldsymbol{D}_{\boldsymbol{\theta}} \dot{\boldsymbol{\theta}} .
\end{aligned}
$$

The latter, being a negative semidefinite function of the state, ensures the stability of $\Omega$.

In order to refine the result (i.e. show that $\Omega$ is asymptotically stable) we will look for the largest invariant set $M$ within the set $E$ defined as ${ }^{2}$

$$
E=\{(\boldsymbol{\theta}, \boldsymbol{q}, \dot{\boldsymbol{\theta}}, \dot{\boldsymbol{q}}) \mid \dot{V}(\boldsymbol{\theta}, \boldsymbol{q}, \dot{\boldsymbol{\theta}}, \dot{\boldsymbol{q}})=0\} .
$$

By virtue of LaSalle's invariance principle, then every solution of the system approaches $M$ as $t \rightarrow \infty$. Since from (11) $\dot{V}(\boldsymbol{\theta}, \boldsymbol{q}, \dot{\boldsymbol{\theta}}, \dot{\boldsymbol{q}})=0 \Leftrightarrow \dot{\boldsymbol{\theta}}=\mathbf{0}$, then starting from the condition $\dot{\boldsymbol{\theta}}=\mathbf{0}$, we will show in two subsequent steps that $M=\left\{\chi_{0}, \Omega\right\}$.

Invariant set: First we show that $\dot{\boldsymbol{\theta}}=\mathbf{0} \Rightarrow \tilde{\boldsymbol{\theta}}=\mathbf{0}$. From $\dot{\boldsymbol{\theta}}=\mathbf{0}$ we can directly conclude that $\boldsymbol{\theta}=\boldsymbol{\theta}_{0}, \boldsymbol{g}(\overline{\boldsymbol{q}}(\boldsymbol{\theta}))=$ $\boldsymbol{g}\left(\overline{\boldsymbol{q}}\left(\boldsymbol{\theta}_{0}\right)\right)=\overline{\boldsymbol{g}}_{0}$ and, because of (10), also $H(\boldsymbol{\theta}, \boldsymbol{q}, \dot{\boldsymbol{\theta}}, \dot{\boldsymbol{q}})=H_{0}$, where $\boldsymbol{\theta}_{0}, \overline{\boldsymbol{g}}_{0}$ and $H_{0}$ are constants. Moreover, from (1b) and (6) we get

$$
-K_{H}\left(H_{0}-H_{d}\right)\left(\tau(\boldsymbol{\phi})-\overline{\boldsymbol{g}}_{0}\right)-\boldsymbol{K}_{\boldsymbol{\theta}}\left(\boldsymbol{\theta}_{0}-\boldsymbol{\theta}_{d}\right)=\mathbf{0},
$$

from which two cases are possible

$$
\left\{\begin{array}{l}
H_{0}=H_{d} \\
H_{0} \neq H_{d}
\end{array} .\right.
$$

While from the first we can directly conclude $\boldsymbol{\theta}=\boldsymbol{\theta}_{d}$, in the second case we get $\tau(\phi)=\tau_{0}$, with $\tau_{0}$ constant. We will now show that the latter condition in turn leads to $\boldsymbol{\theta}=\boldsymbol{\theta}_{d}$. From the definition of $\tau(\phi)$ and the fundamental theorem of calculus for line integrals, we get

$$
U_{k}\left(\phi_{1}\right)-U_{k}\left(\phi_{2}\right)=\tau_{0}^{T}\left(\phi_{1}-\phi_{2}\right)
$$

\footnotetext{
${ }^{2}$ As we will see later, this set coincides with the set $E$ in Lemma 1. This justifies the use of the same letter.
}

which holds, without contradicting assumption (A1), $\forall \phi_{1}, \phi_{2} \in \mathbb{R}^{n}$ if and only if $\phi_{1}=\phi_{2}$. This implies $\phi=\phi_{0}$ and $\boldsymbol{q}=\boldsymbol{q}_{0}$, where $\boldsymbol{\phi}_{0}$ and $\boldsymbol{q}_{0}$ are constants. Consequently, from (1a), we get $\boldsymbol{g}\left(\boldsymbol{q}_{0}\right)=\boldsymbol{\tau}_{0}$ and from the uniqueness of (3) that $\boldsymbol{q}_{0}=\overline{\boldsymbol{q}}\left(\boldsymbol{\theta}_{0}\right)$. Concluding, if $H_{0} \neq H_{d}$ then $\boldsymbol{\tau}(\boldsymbol{\phi})=\overline{\boldsymbol{g}}_{0}$ and therefore also in this case $\boldsymbol{\theta}=\boldsymbol{\theta}_{d}$.

Now we finally prove that $\dot{\boldsymbol{\theta}}=\tilde{\boldsymbol{\theta}}=\mathbf{0} \Rightarrow M=\left\{\chi_{0}, \Omega\right\}$. In fact, in addition to the previous conditions we have $\boldsymbol{\theta}=\boldsymbol{\theta}_{d}$, therefore from (1b) and (6) it follows that

$$
-K_{H}\left(H_{0}-H_{d}\right)\left(\boldsymbol{\tau}\left(\boldsymbol{\theta}_{d}-\boldsymbol{q}\right)-\boldsymbol{g}\left(\overline{\boldsymbol{q}}\left(\boldsymbol{\theta}_{d}\right)\right)\right)=\mathbf{0} .
$$

If $H_{0}=H_{d}$ we get $\Omega$ by definition, while in case $H_{0} \neq H_{d}$ then $\boldsymbol{\tau}\left(\boldsymbol{\theta}_{d}-\boldsymbol{q}\right)=\boldsymbol{g}\left(\overline{\boldsymbol{q}}\left(\boldsymbol{\theta}_{d}\right)\right)$, that is $\boldsymbol{\tau}(\boldsymbol{\phi})$ is constant and, as it has been shown before, this implies that $\boldsymbol{q}=\overline{\boldsymbol{q}}\left(\boldsymbol{\theta}_{d}\right), \dot{\boldsymbol{q}}=\mathbf{0}$ or in other words $(\boldsymbol{\theta}, \boldsymbol{q}, \dot{\boldsymbol{\theta}}, \dot{\boldsymbol{q}})=\chi_{0}$.

Asymptotic stability: Since $M$ does not contain only $\Omega$, we still cannot conclude that $\Omega$ is asymptotically stable. Nevertheless, let us first consider the case $H_{d}=0$. Using (5) it can be easily verified that the positively invariant set $\Omega$ coincides with $\chi_{0}$, which becomes the only positive limit set and therefore result $(a)$ is obtained. On the other hand, when $H_{d}>0$ we can apply Lemma 1 to show that the equilibrium point $\chi_{0}$ is unstable. This means that all the solutions, except for the trivial one $(\boldsymbol{\theta}(t), \boldsymbol{q}(t), \dot{\boldsymbol{\theta}}(t), \dot{\boldsymbol{q}}(t))=\chi_{0}, \forall t \geq 0$, will converge to $\Omega$ as $t \rightarrow \infty$. To this end, the scalar function $W$ is chosen as $W(\boldsymbol{\theta}, \boldsymbol{q}, \dot{\boldsymbol{\theta}}, \dot{\boldsymbol{q}})=-V(\boldsymbol{\theta}, \boldsymbol{q}, \dot{\boldsymbol{\theta}}, \dot{\boldsymbol{q}})$. In this way, for the closed loop system, $\dot{W}(\boldsymbol{\theta}, \boldsymbol{q}, \dot{\boldsymbol{\theta}}, \dot{\boldsymbol{q}}) \geq 0$ and the set $M$, which is the intersection of the positive limit set of the solutions and the set

$$
\begin{aligned}
E & =\{(\boldsymbol{\theta}, \boldsymbol{q}, \dot{\boldsymbol{\theta}}, \dot{\boldsymbol{q}}) \mid \dot{V}(\boldsymbol{\theta}, \boldsymbol{q}, \dot{\boldsymbol{\theta}}, \dot{\boldsymbol{q}})=0\} \\
& =\{(\boldsymbol{\theta}, \boldsymbol{q}, \dot{\boldsymbol{\theta}}, \dot{\boldsymbol{q}}) \mid \dot{W}(\boldsymbol{\theta}, \boldsymbol{q}, \dot{\boldsymbol{\theta}}, \dot{\boldsymbol{q}})=0\} .
\end{aligned}
$$

contains no stable equilibrium points. In case $n=1$ the set $\Omega$ is a closed orbit in the state space and therefore a limit cycle, proving result $(b)$. Namely, decreasing the value of $H_{d}$, the asymptotically stable limit cycle collides on the unstable equilibrium point, originating an asymptotically stable equilibrium point.

\section{A. Controller discussion}

The key feature of the proposed controller is in a torque feedback that, depending on the energy error, can be either positive or negative and therefore intuitively able to either inject or dissipate energy. The gain of the feedback can be additionally tuned if a generalization of the control law is used

$\boldsymbol{u}=\boldsymbol{\tau}(\boldsymbol{\phi})-K_{H} \tilde{H}^{m-1}(\boldsymbol{\theta}, \boldsymbol{q}, \dot{\boldsymbol{\theta}}, \dot{\boldsymbol{q}})(\boldsymbol{\tau}(\boldsymbol{\phi})-\boldsymbol{g}(\overline{\boldsymbol{q}}(\boldsymbol{\theta})))-\boldsymbol{K}_{\boldsymbol{\theta}} \tilde{\boldsymbol{\theta}}-\boldsymbol{D}_{\boldsymbol{\theta}} \dot{\boldsymbol{\theta}}$,

obtained using the more general term $\frac{1}{m} K_{H} \tilde{H}^{m}(\boldsymbol{\theta}, \boldsymbol{q}, \dot{\boldsymbol{\theta}}, \dot{\boldsymbol{q}})$ in the Lyapunov function, with $m=2 k, k \in \mathbb{N}^{+}$. Obviously, the case examined so far is retrieved when $k=1$.

When used to produce an oscillation, the proposed controller is expected to be very efficient. As it was shown, after a transient in which the motors bring the energy to the desired level no more power is provided by them since the velocity goes to zero. Basically, the oscillation will be sustained by 
the springs indefinitely. Obviously, this can happen only in the nominal case while in reality there will be always friction taking out energy that the motors will have to inject again in the system.

Concerning the computational cost of the proposed control law, from (6) it is clear that the mass matrix $\boldsymbol{M ( q )}$ and the total potential energy need to be computed in order to evaluate $H(\boldsymbol{\theta}, \boldsymbol{q}, \dot{\boldsymbol{\theta}}, \dot{\boldsymbol{q}})$. In addition, the gradient of the potential functions and the function $\overline{\boldsymbol{q}}$ are required. The latter can be computed using a fixed-point iteration or Newton methods to solve (3), as shown in [9] where also comments concerning the efficiency of the approach can be found. Finally, the Coriolis and centrifugal terms which are the most costly are not required, as well as no higher order differentiation of any of the involved expressions, which are required in many nonlinear controllers, e.g. those based on feedback linearization.

\section{VALIDATION}

In this section we show the results obtained when applying the controller proposed in Theorem 1 to the robotic system in Fig. 1, both in simulation and experiments. The DLR Hand Arm System (HASy) [12] is a highly integrated robot, which aims to imitate the whole human upper limb in terms of size, weight and range of motion. Variable stiffness actuators (VSA), whose intrinsic nonlinear compliance can be adjusted by an additional motor, are used to replace the typically impedance controlled, mechanically stiff joints. As a result the robot can, for example, better handle collisions with stiff objects since part of the impact energy, which changes too quickly for an active controller, is temporarily stored in the elastic components. The system has more than 25 degrees of freedom, 50 motors and 100 position sensors. In our setup we will not adjust the stiffness on line so that the system dynamics can be described as in (1). Moreover, we profit of the possibility of using only the upper arm to focus our attention on the first four joints (which share the same mechanical design), as indicated in Fig. 1.

In order to show the behavior of our control law, we will always conduct two tests. In the first one, we induce an oscillation in the first joint while the motion is damped out with a classic PD control in the remaining ones. In particular the axis are oriented in such a way that the effect of the gravity are negligible and therefore the dynamic coupling between the joints can be approximately compensated with a high gain motor PD controller. In the other, we use our control law to regulate the robot to a desired configuration, which corresponds to ask for zero energy in the system. The two tests will always be labeled as respectively $(a)$ and $(b)$ in all the figures. Fig. 1 sums up the first test, highlighting the joints involved and their role. In addition, a conceptual interpretation of what happens in the state space is provided. Imagining to have a three dimensional state space, the set $\Omega$ of constant desired energy can, for example, be thought as a sphere where the close loop system is bound to evolve. The additional constraints introduced through the PD controller, render one of the infinite many possible iso-energy orbits the

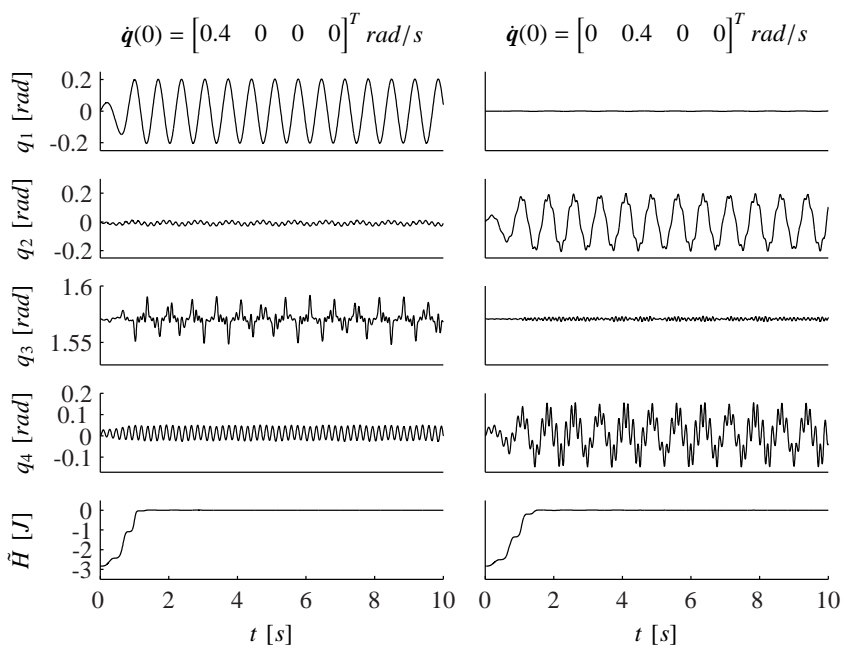

Fig. 2. Link positions obtained applying our controller to all the joints with $H_{d}=3 \mathrm{~J}$. Each column refers to a trial, while along the rows the same variable in the two different tests is shown. The same scale is used to highlight the differences. The two trials only differ for the value of the initial link side velocities. Although the same set $\Omega$ is reached, the resulting orbit in the state space is obviously different.

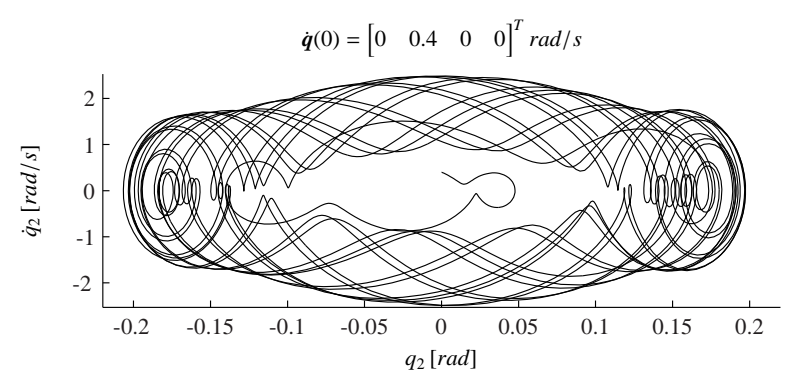

Fig. 3. Visualization of the chaotic behavior through the phase plot of the link side position and velocity of the second joint. The solution does not keep following the same trajectory as it should happen in case of a period motion.

one actually followed by the system. Basically the system can be thought as a one joint robot for which the existence of an asymptotically stable limit cycle has been proved. Fig. 2 supports the explanation. There the link positions of two trials obtained applying the control law (6) to all the joints of the robot with a strictly positive desired energy is shown. The only difference between the two trials is in the initial value of the link velocities. Even though in both cases the same set $\Omega$ is reached, as it can be seen the orbit followed by the system is different. In particular the oscillation produce in this case is the consequence of a chaotic behavior and not a periodic one, as the phase plot of the link variables of the second joint shows in Fig. 3.

\section{A. Simulations}

The simulations are carried out using MATLAB/Simulink ${ }^{\circledR}$, where the algorithm and formulas from [17] are implemented to compute all the quantities necessary for the control law and the robot direct dynamics. In addition, in this section, all the plots will show with a blue line the performances of the controller in presence of 
(a)
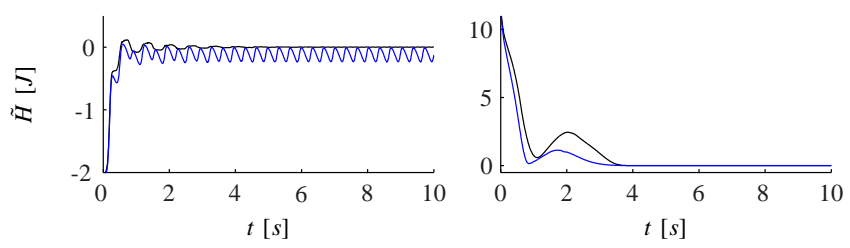

Fig. 4. Convergence of the error $H(\boldsymbol{\theta}, \boldsymbol{q}, \dot{\boldsymbol{\theta}}, \dot{\boldsymbol{q}})-H_{d}$. Both for limit cycle generation (case $(a)$ ) and damping of the oscillations (case $(b)$ ) the error always converges to zero, the different behavior is only due to the value of $H_{d}$. The blue line shows different performance in presence of a link side friction unknown to the controller.

a non modeled link side friction $-d \dot{\boldsymbol{q}}$, with $d=0.5 \frac{\mathrm{N} \cdot \mathrm{m} \cdot \mathrm{s}}{\mathrm{rad}}$. While this term is of course not harmful when the system is required to damp out the oscillations, it forces the controller to re-inject the lost energy due to the unforeseen energy leak. This is especially clear in Fig. 4 where the convergence of the error $\tilde{H}(\boldsymbol{\theta}, \boldsymbol{q}, \dot{\boldsymbol{\theta}}, \dot{\boldsymbol{q}})=H(\boldsymbol{\theta}, \boldsymbol{q}, \dot{\boldsymbol{\theta}}, \dot{\boldsymbol{q}})-H_{d}$ is shown, both for damping of the oscillations and limit cycle generation. The different behavior of the system is simply due to a different value of $H_{d}$, which is $H_{d}=3 \mathrm{~J}$ for limit cycle generation and $H_{d}=-1 \mathrm{~J}$ for regulation to the desired configuration ${ }^{3}$. Concerning the value of the other parameters, the gains are chosen as

$$
\begin{aligned}
& \text { - } K_{H}=5 \frac{1}{\mathrm{~J}} \\
& \text { - } \boldsymbol{K}_{\boldsymbol{\theta}}=\operatorname{diag}\left(\left[\begin{array}{llll}
20 & 20 & 20 & 20
\end{array}\right]\right) \frac{\mathrm{N} \cdot \mathrm{m}}{\mathrm{rad}} \\
& \text { - } \boldsymbol{D}_{\boldsymbol{\theta}}=\operatorname{diag}\left(\left[\begin{array}{llll}
20 & 20 & 20 & 20
\end{array}\right]\right) \frac{\mathrm{N} \cdot \mathrm{m} \cdot \mathrm{s}}{\mathrm{rad}}
\end{aligned}
$$

when the robot is controlled to the desired configuration, while

$$
\begin{aligned}
& \text { - } K_{H}=0.5 \frac{1}{\mathrm{~J}} \\
& \text { - } K_{\theta}=20 \frac{\mathrm{N} \cdot \mathrm{m}}{\mathrm{rad}} \\
& \text { - } D_{\theta}=20 \frac{\mathrm{N} \cdot \mathrm{m} \cdot \mathrm{s}}{\mathrm{rad}}
\end{aligned}
$$

in case an oscillation is required to be induced. In all the simulations the desired value of the motor position is $\boldsymbol{\theta}_{d}=\left[\begin{array}{llll}0 & 0 & \pi / 2 & 0\end{array}\right]^{T} \mathrm{rad}$, as it can be seen in Fig. 5 for the two tests. It is worth to mention that the robot is always initialized in the equilibrium position, but with a nonzero initial link side velocity. The latter is always chosen to be $2 \mathrm{rad} / \mathrm{s}$ for the joints where the control law (6) is active, i.e. only the first joint in case of excitation and all the joints in case of damping of the oscillations. This explains why in Fig. 5, after a transient, the motors recover their initial positions. Nevertheless, in case $H_{d}>0$, a very small oscillation is present in the joints controlled with the simple PD law. This is not surprising since we are tying to make a multi-joints system behave like a single joint one. For the same reason also the link side positions will have a coupled oscillation and will qualitatively look like the ones in Fig. 2, case $\dot{\boldsymbol{q}}(0)=\left[\begin{array}{llll}2 & 0 & 0 & 0\end{array}\right]^{T} \mathrm{rad} / \mathrm{s}$. To conclude, in Fig. 6

\footnotetext{
${ }^{3}$ Asking for a negative desired value for $H(\boldsymbol{\theta}, \boldsymbol{q}, \dot{\boldsymbol{\theta}}, \dot{\boldsymbol{q}})$ has the only effect to increase the convergence speed and reduce residual small oscillations around the desired value due to numeric errors. The smallest possible value for $H(\boldsymbol{\theta}, \boldsymbol{q}, \dot{\boldsymbol{\theta}}, \dot{\boldsymbol{q}})$ is zero, which is the one that will be reached. In Fig. $4(b)$ the plot is shifted of $1 \mathrm{~J}$ to take this effect into account.
}

(a)
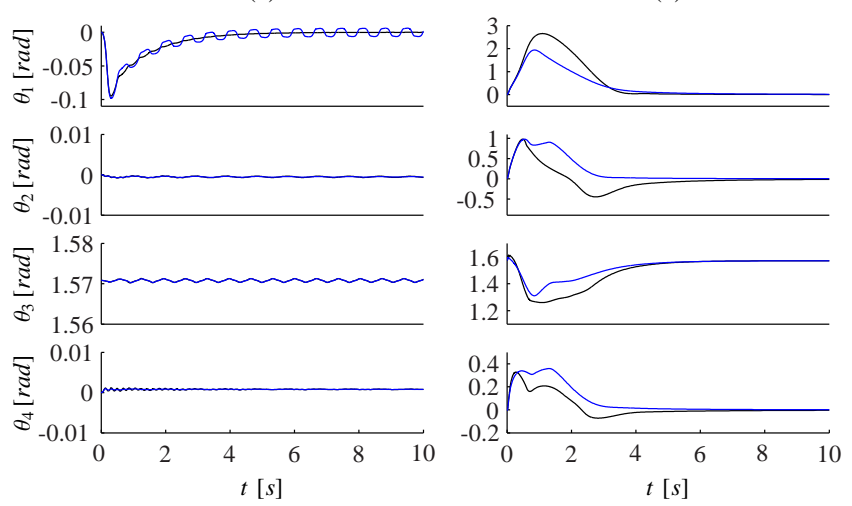

Fig. 5. The left column (a) collects the motor positions in case of limit cycle generation, the right $(b)$ in case the oscillation is damped out. Along the rows the motor position of the same joint in the two cases is shown. Even though the robot starts from the desired configuration, the initial non zero link side velocity acts as a disturbance, which is recovered after the transient. The blue line shows different performance in presence of a link side friction unknown to the controller.


Fig. 6. Link position and velocities when an initial velocity perturbs the robot from the equilibrium point. Using $H_{d}=-1 \mathrm{~J}$, after a short transient, the system evolution brings back the robot to its initial state. The blue line shows different performance in presence of a link side friction unknown to the controller.

the link positions, as well as the velocities, are shown from which it can be seen that the initial oscillations are damped out using a non positive desired value for $H(\boldsymbol{\theta}, \boldsymbol{q}, \dot{\boldsymbol{\theta}}, \dot{\boldsymbol{q}})$.

\section{B. Experiments}

Using the same values of the parameters as in the previous subsection, we conducted two tests with the real system. In the first one, the system is initialized in the equilibrium point and we choose $H_{d}=3 \mathrm{~J}$. Fig. 7 shows the "linkside energy" (black line) and its desired value (blue line). When the operator slightly pushes the robot, it starts moving away from the equilibrium point and oscillating around the desired value of the energy. As expected, the system does not exactly converge to the desired value, but it exhibits a behavior similar to the one observed in simulation in case of unmodeled friction. Of course, due to noise and bigger model uncertainty than the simulated case, we also observe a bigger deviation from the desired value. When the desired 


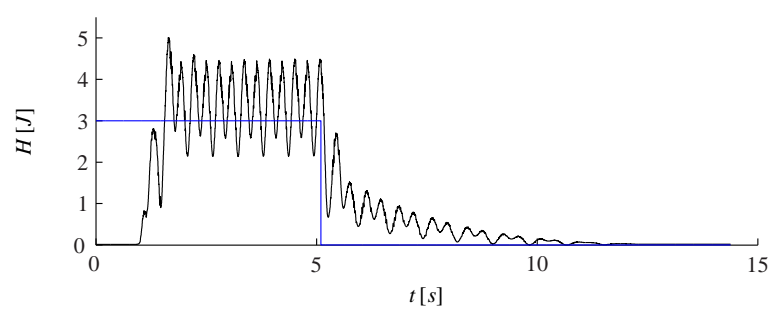

Fig. 7. "Link-side energy" $H(\boldsymbol{\theta}, \boldsymbol{q}, \dot{\boldsymbol{\theta}}, \dot{\boldsymbol{q}})$ (black line) and its desired value (blue line).

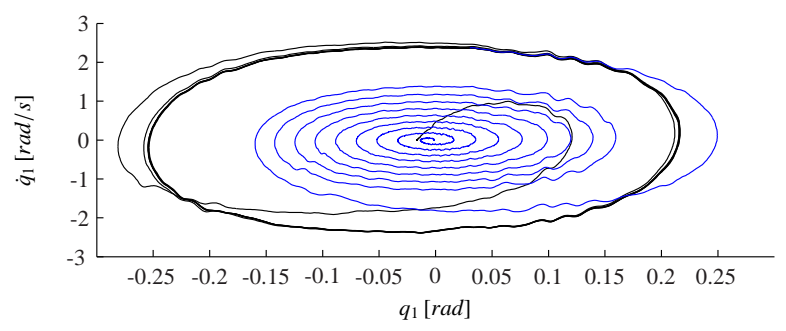

Fig. 8. Phase plot of the link side position and velocity of the first joint. The value of $H_{d}$ is switched on-line from $H_{d}=3 \mathrm{~J}$ (black line) to $H_{d}=0 \mathrm{~J}$ (blue line), causing the system to move from the limit cycle to the equilibrium point.

value is switched to zero, the energy converges to it without any residual oscillation.

Particularly interesting is the phase plot of the link variables involved in the generation of the oscillation, shown in Fig. 8. There with a black line is shown the behavior when $H_{d}=3 \mathrm{~J}$ and with a blue line when $H_{d}=0 \mathrm{~J}$. As it can be seen, despite the presence of noise and model uncertainty, the system does reach a limit cycle. Basically what will be perturbed is the shape of the limit cycle itself. Adaptive techniques and tuning of the parameters can of course reduce these effects. The spiraling convergence towards the asymptotically stable equilibrium point obtained when the desired energy is set to zero is also shown in the same plot. It is worth to mention that the damping behavior of the system can be tuned through $H_{d}$ and $K_{H}$, since they will influence how quickly $H(\boldsymbol{\theta}, \boldsymbol{q}, \dot{\boldsymbol{\theta}}, \dot{\boldsymbol{q}})$ will converge to zero and therefore how quickly the link side oscillations will be damped out.

In Fig. 9 both the motor and link positions of the first joint are shown. It is interesting to notice that, although the motor is not fixed because of friction, the oscillations obtained on the link side are still amplified compared to those required from the motor.

In the second test ${ }^{4}$ all the joints are involved and we analyze the response of the system to a step in the desired value of the motor positions. These are reported in Fig. 10, where the blue lines show the desired values and the black lines the real motor positions. As it can be noted, the systems

\footnotetext{
${ }^{4}$ In this experiment to show the high achievable performances of the system we use $\boldsymbol{K}_{\boldsymbol{\theta}}=\operatorname{diag}\left(\left[\begin{array}{llll}400 & 400 & 400 & 400\end{array}\right]\right) \frac{\mathrm{N} \cdot \mathrm{m}}{\mathrm{rad}}$.
}
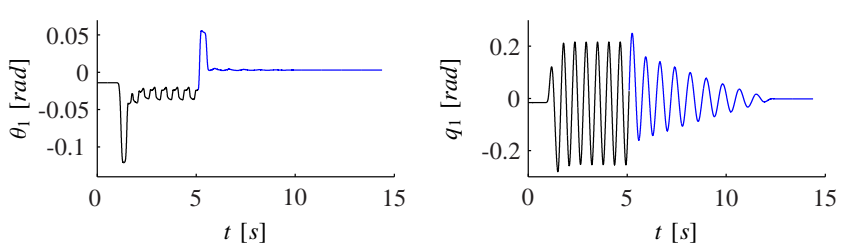

Fig. 9. Motor and link positions of the first joint when $H_{d}$ is switched from $H_{d}=3 \mathrm{~J}$ (black line) to $H_{d}=0 \mathrm{~J}$ (blue line).
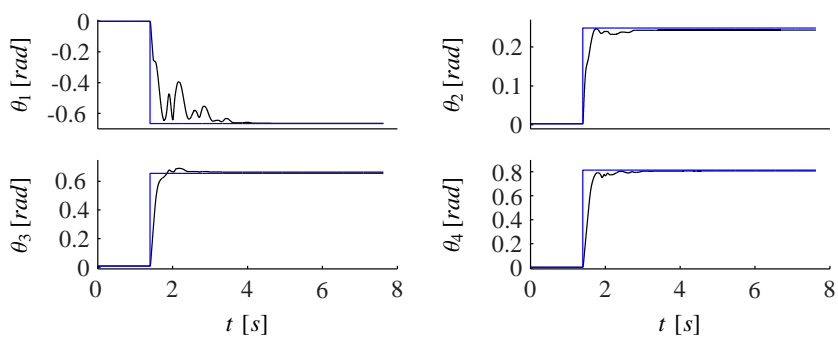

Fig. 10. Motor positions (black lines) and desired values (blue lines). Using $H_{d}=0 \mathrm{~J}$ the system moves from one equilibrium point to the new one defined by the new value of $\boldsymbol{\theta}_{d}$.

shows a good convergence behavior.

Concerning the link side, in Fig. 11 the link velocities are shown which prove that the robot can reach the new desired equilibrium point and no unwanted residual oscillations of the links are present.

\section{Conclusion}

In this paper a new control law for elastic joint robots has been proposed. It allows, by simply changing the desired value of an "energy-like" function, for both regulation to a desired configuration of the robot and generation of oscillations. The last ones are produced by the elastic elements present in the joints themselves, therefore efficiency can be achieved. It is clear, in fact, that being the motor velocities zero once the energy reaches the desired value, no more power is provided by the motors. Moreover, it has been proven that the oscillation is due to the presence of an asymptotically stable limit cycle in the state space of the closed loop system, if the robot has only one joint. Further investigations to obtain a limit cycle also for a multi-joints robot are currently under consideration, in order to extend
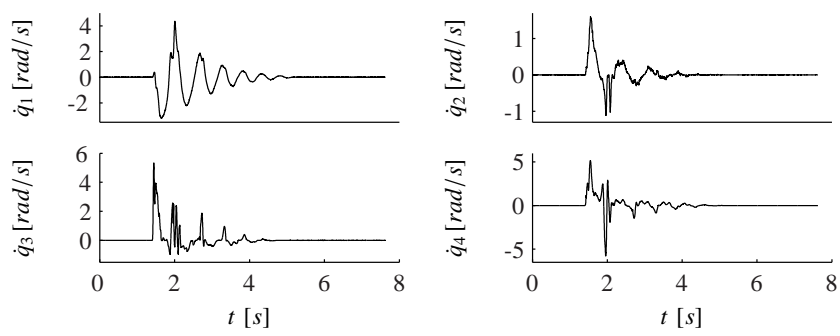

Fig. 11. Link velocities obtained after a step in the desired motor position, with $H_{d}=0 \mathrm{~J}$. After a transient also the links reach the new desired configuration with zero velocity. 
the results of our previous work [2] from rigid to elastic joint robots.

\section{APPENDIX}

\section{TOTAL POTENTIAL}

The result presented here can be directly applied to prove (5). In particular we follow the idea used in [10] not only for the gravitational potential, but also for the elastic potential, in such a way that an upper bound for the total potential can be found.

Lemma 2: Given assumptions (A2), (A3) the total potential function $U(\boldsymbol{\theta}, \boldsymbol{q})=U_{g}(\boldsymbol{q})+U_{k}(\boldsymbol{\theta}-\boldsymbol{q})$, satisfies the conditions

$$
\begin{cases}U(\boldsymbol{\theta}, \boldsymbol{q}) \geq U(\boldsymbol{\theta}, \overline{\boldsymbol{q}}(\boldsymbol{\theta})) & \forall \boldsymbol{\theta}, \boldsymbol{q} \in \mathbb{Q} \\ U(\boldsymbol{\theta}, \boldsymbol{q})=U(\boldsymbol{\theta}, \overline{\boldsymbol{q}}(\boldsymbol{\theta})) & \boldsymbol{q}=\overline{\boldsymbol{q}}(\boldsymbol{\theta}), \boldsymbol{\theta} \in \mathbb{Q} .\end{cases}
$$

Proof: Given any potential function $U_{0}(\chi)$ it is always possible to write

$$
U_{0}\left(\chi_{2}\right)-U_{0}\left(\chi_{1}\right)-\left.\frac{\partial U_{0}(\chi)}{\partial \chi}\right|_{\chi_{1}}\left(\chi_{2}-\chi_{1}\right)=i_{0}\left(\chi_{1}, \chi_{2}\right)
$$

where $i_{0}\left(\chi_{1}, \chi_{2}\right)=\int_{\chi_{1}}^{\chi_{2}}\left(\left.\int_{\chi_{1}}^{\chi} \frac{\partial^{2} U_{0}(\chi)}{\partial \chi^{2}}\right|_{\xi} \mathrm{d} \xi\right)^{T} \mathrm{~d} \chi$.

Moreover, since $i_{0}\left(\chi_{1}, \chi_{2}\right)$ is path independent, we can choose in particular the paths $\xi(\lambda):[0,1] \rightarrow \mathbb{Q}$ and $\chi(\gamma):$ $[0,1] \rightarrow \mathbb{Q}$

$$
\begin{aligned}
& \xi(\lambda):=\chi_{1}+\gamma\left(\chi-\chi_{1}\right) \\
& \chi(\gamma):=\chi_{1}+\lambda\left(\chi_{2}-\chi_{1}\right)
\end{aligned}
$$

such that evaluating the integral along them we obtain

$$
\left.\int_{0}^{1} \int_{0}^{1}\left(\chi_{2}-\chi_{1}\right)^{T} \frac{\partial^{2} U_{0}(\chi)}{\partial \chi^{2}}\right|_{\chi_{1}+\gamma \lambda\left(\chi_{2}-\chi_{1}\right)}\left(\chi_{2}-\chi_{1}\right) \gamma \mathrm{d} \lambda \mathrm{d} \gamma
$$

If $U_{0}(\chi)=U_{g}(\boldsymbol{q})$, we can take the absolute value and use Proposition 1 to derive the following inequalities

$$
\begin{aligned}
\left|i_{g}\left(\boldsymbol{q}_{1}, \boldsymbol{q}_{2}\right)\right| & \leq \int_{0}^{1} \int_{0}^{1} \sup _{\forall \boldsymbol{q} \in \mathbb{Q}}\left\|\frac{\partial^{2} U_{g}(\boldsymbol{q})}{\partial \boldsymbol{q}^{2}}\right\|\left\|\boldsymbol{q}_{2}-\boldsymbol{q}_{1}\right\|^{2} \gamma \mathrm{d} \lambda \mathrm{d} \gamma \\
& <\frac{1}{2} \beta\left\|\boldsymbol{q}_{2}-\boldsymbol{q}_{1}\right\|^{2},
\end{aligned}
$$

while if $U_{0}(\chi)=U_{k}(\phi)$, using assumption (A2) it follows that

$$
\frac{1}{2} \alpha_{1}\left\|\boldsymbol{\phi}_{2}-\boldsymbol{\phi}_{1}\right\|^{2}<i_{\psi}\left(\boldsymbol{\phi}_{1}, \boldsymbol{\phi}_{2}\right)<\frac{1}{2} \alpha_{2}\left\|\boldsymbol{\phi}_{2}-\boldsymbol{\phi}_{1}\right\|^{2}
$$

where $i_{g}$ and $i_{\psi}$ are to be considered as the function $i_{0}$ with the correspondent potential function in it. Using $i_{g}(\overline{\boldsymbol{q}}(\boldsymbol{\theta}), \boldsymbol{q})$ and $i_{\psi}(\boldsymbol{\theta}-\overline{\boldsymbol{q}}(\boldsymbol{\theta}), \boldsymbol{\theta}-\boldsymbol{q})$ the following holds

$$
\begin{array}{r}
U(\boldsymbol{\theta}, \boldsymbol{q})-U(\boldsymbol{\theta}, \overline{\boldsymbol{q}}(\boldsymbol{\theta}))=i_{g}(\overline{\boldsymbol{q}}(\boldsymbol{\theta}), \boldsymbol{q})+i_{\psi}(\boldsymbol{\theta}-\overline{\boldsymbol{q}}(\boldsymbol{\theta}), \boldsymbol{\theta}-\boldsymbol{q})+ \\
(\boldsymbol{g}(\overline{\boldsymbol{q}}(\boldsymbol{\theta}))-\boldsymbol{\tau}(\boldsymbol{\theta}-\overline{\boldsymbol{q}}(\boldsymbol{\theta})))^{T}(\boldsymbol{q}-\overline{\boldsymbol{q}}(\boldsymbol{\theta})),
\end{array}
$$

which leads to the inequality

$$
U(\boldsymbol{\theta}, \boldsymbol{q})-U(\boldsymbol{\theta}, \overline{\boldsymbol{q}}(\boldsymbol{\theta}))<\frac{1}{2}\left(\alpha_{1}-\beta\right)\|\boldsymbol{q}-\overline{\boldsymbol{q}}(\boldsymbol{\theta})\|^{2},
$$

if (3), (19) and (20) are used. From the last inequality and assumption (A3) the conditions on the total potential function $U(\boldsymbol{\theta}, \boldsymbol{q})$ follow directly, since $\alpha_{1}-\beta>0$.

\section{ACKNOWLEDGMENT}

This research is partly supported by the Initiative and Networking Fund of the Helmholtz Association through a Helmholtz Young Investigators Group (Grant no. VH-NG808).

\section{REFERENCES}

[1] G. Garofalo, C. Ott, and A. Albu-Schäffer, "Walking control of fully actuated robots based on the bipedal slip model," in IEEE Int. Conf. on Robotics and Automation (ICRA), Saint Paul, USA, May 2012, pp. 1999-2004.

[2] - "Orbital stabilization of mechanical systems through semidefinite Lyapunov functions," in American Control Conference (ACC), Washington DC, USA, June 2013.

[3] D. Lakatos, G. Garofalo, F. Petit, C. Ott, and A. Albu-Schäffer, "Modal limit cycle control for variable stiffness actuated robots," in IEEE Int. Conf. on Robotics and Automation (ICRA), Karlsruhe, Germany, May 2013, pp. 4934-4941.

[4] T. Petric, L. Zlajpah, G. Garofalo, and C. Ott, "Walking control using adaptive oscillators combined with dynamic movement primitives," in 22nd International Workshop on Robotics in Alpe-Adria-Danube Region (RAAD), PortoroA, Slovenia, Sept. 2013, pp. 204-211.

[5] D. Lakatos, G. Garofalo, A. Dietrich, and A. Albu-Schäffer, "Jumping control for compliantly actuated multilegged robots," in IEEE Int. Conf. on Robotics and Automation (ICRA), Hong Kong, China, May 2014.

[6] A. De Luca, "Dynamic control of robots with joint elasticity," in IEEE Int. Conf. on Robotics and Automation (ICRA), Philadelphia, USA, Apr. 1988, pp. 152-158.

[7] S. Nicosia and P. Tomei, "On the feedback linearization of robots with elastic joints," in IEEE Conf. on Decision and Control, Austin, USA, Dec. 1988, pp. 180-185.

[8] M. Garabini, A. Passaglia, F. Belo, P. Salaris, and A. Bicchi, "Optimality principles in variable stiffness control: The VSA Hammer," in IEEE/RSJ Int. Conf. on Intelligent Robots and Systems (IROS), San Francisco, USA, Sept. 2011, pp. 3770-3775.

[9] A. Albu-Schäffer, C. Ott, and F. Petit, "Energy shaping control for a class of underactuated Euler-Lagrange systems," in Proc. 10th Int. IFAC Symp. Robot Control, Dubrovnik, Croatia, Sept. 2012, pp. $567-$ 575.

[10] C. Ott, Cartesian Impedance Control of Redundant and Flexible-Joint Robots, ser. Springer Tracts in Advanced Robotics. Berlin: SpringerVerlag, 2008.

[11] F. Petit, C. Ott, and A. Albu-Schäffer, "A model-free approach to vibration suppression for intrinsically elastic robots," in IEEE Int. Conf. on Robotics and Automation (ICRA), Hong Kong, China, May 2014.

[12] M. Grebenstein, A. Albu-Schäffer, T. Bahls, M. Chalon, O. Eiberger, W. Friedl, R. Gruber, S. Haddadin, U. Hagn, R. Haslinger, H. Höppner, S. Jörg, M. Nick1, A. Nothhelfer, F. Petit, J. Reill, N. Seitz, T. Wimböck, S. Wolf, T. Wüsthoff, and G. Hirzinger, "The DLR Hand Arm System," in IEEE Int. Conf. on Robotics and Automation (ICRA), Shanghai, China, May 2011, pp. 3175-3182.

[13] M. W. Spong, "Modeling and control of elastic joint robots," the ASME Journal of Dynamic Systems, Measurement, and Control, vol. 109, pp. 310-318, 1987.

[14] C. Canudas-de-Wit, B. Siciliano, and G. Bastin, Theory of robot control. London: Springer-Verlag, 1996.

[15] A. Arsie and C. Ebenbauer, "Refining LaSalle's invariance principle," in American Control Conference (ACC), Saint Louis, USA, June 2009, pp. $108-112$.

[16] H. K. Khalil, Nonlinear Systems. New Jersey: Prentice Hall, 2002.

[17] G. Garofalo, C. Ott, and A. Albu-Schäffer, "On the closed form computation of the dynamic matrices and their differentiations," in IEEE/RSJ Int. Conf. on Intelligent Robots and Systems (IROS), Tokyo, Japan, Nov. 2013. 